\title{
Mechanical input restrains PPARy2 expression and action to preserve mesenchymal stem cell multipotentiality
}

\author{
Natasha Case ${ }^{\mathrm{a}}$, Jacob Thomas ${ }^{\mathrm{a}}$, Zhihui Xie ${ }^{\mathrm{a}}$, Buer Sen ${ }^{\mathrm{a}}$, Maya Stynera ${ }^{\mathrm{a}}$ David Rowe $^{\mathrm{b}}$, and \\ Janet Rubin ${ }^{\mathrm{a}}$ \\ Jacob Thomas: jacobt@email.unc.edu; Zhihui Xie: zhihui_xie@med.unc.edu; Buer Sen: bsen@med.unc.edu; Maya \\ Styner: mstyner@med.unc.edu; David Rowe: rowe@neuron.uchc.edu; Janet Rubin: jrubin@med.unc.edu \\ aDepartment of Medicine, University of North Carolina, 125 MacNider Hall, CB\# 7005, Chapel \\ Hill, NC 27599 U.S.A \\ bDepartment of Reconstructive Sciences, University of Connecticut Health Center, 263 \\ Farmington Avenue, Farmington, Connecticut 06030 U.S.A
}

\section{Abstract}

Exercise-generated signals are pro-osteogenic and anti-adipogenic within the marrow. In vitro studies indicate that mechanical signals directly block adipogenic differentiation through activation of $\beta$-catenin and by limiting PPAR $\gamma 2$ expression. Whether mechanically generated $\beta$ catenin can inhibit adipogenesis during PPAR $\gamma$ transactivation is unknown. We evaluated the ability of mechanical signals to limit adipogenesis in marrow derived mesenchymal stem cells (mdMSC) distal to activation of PPAR $\gamma$. First, we established that mdMSC attained an adipogenic phenotype within 2-4 days in the presence of rosiglitazone (1-25 $\mu \mathrm{M})$ and that $\beta$-catenin activation via GSK3 $\beta$ inhibition interfered with this process. Similarly, mechanical strain (3600 cycles, $2 \%$ strain daily) inhibited adipogenesis at 3 days, preventing rosiglitazone-induced PPAR $\gamma$ upregulation as well as aP2 and adiponectin protein expression. To assess whether a reduction in PPAR $\gamma$ expression was necessary for anti-adipogenic action, PPAR $\gamma 2$ was overexpressed: both mechanical strain and GSK3 $\beta$ inhibition prevented expression of aP 2 and adiponectin proteins despite abundant PPAR $\gamma 2$ and its ligand. To understand the fate of single cells experiencing mechanical strain we generated mdMSC from aP2-GFP reporter expressing mice. Rosiglitazone treatment for 3 days induced GFP expression in more than $80 \%$ of cells. Sorting by GFP expression revealed that the highest $20 \%$ of aP2-GFP expressing cells was responsible for the majority of adipogenic protein expression. This highly expressing GFP fraction had a reduced ability to respond to an osteogenic stimulus: BMP-2 treatment increased osterix by 12 -fold in contrast to the 42-fold increase in osterix expression that resulted from BMP-2 treatment of the bottom 75\% of GFP expressing cells. This suggested that highly expressing aP2-GFP cells represented more terminally differentiated adipocytes, with reduced multipotentiality. Application of mechanical strain to aP2-GFP mdMSC treated with rosiglitazone caused a two-fold decrease in the size of the upper cell fraction, suggesting that mechanical strain preserved MSC in a multipotent state. Our data show that mechanical strain restricts adipogenesis both by limiting PPAR $\gamma 2$ expression and by preventing PPAR $\gamma$ action, protecting the potential of MSC to enter other lineages.

(C) 2012 Elsevier Inc. All rights reserved.

Corresponding Author: Natasha Case, PhD, 5030 Burnett-Womack, CB 7170, UNC@Chapel Hill, Chapel Hill, NC 27599. Tel.: 919.966.6743; Fax: 919.966.3338; ncase@med.unc.edu.

Publisher's Disclaimer: This is a PDF file of an unedited manuscript that has been accepted for publication. As a service to our customers we are providing this early version of the manuscript. The manuscript will undergo copyediting, typesetting, and review of the resulting proof before it is published in its final citable form. Please note that during the production process errors may be discovered which could affect the content, and all legal disclaimers that apply to the journal pertain. 


\section{Keywords}

adipogenesis; bone marrow; rosiglitazone; aP2; $\beta$-catenin

\section{Introduction}

Skeletal health over the lifetime of an organism is critically influenced by the function of the mesenchymal and hematopoietic stem cell populations, which give rise to osteoblasts and osteoclasts, respectively. MSC function, in terms of self-renewal and the ability to differentiate into the osteogenic lineage, declines with aging [1], coincident with the progression of osteoporosis. Weight-bearing exercise is beneficial to skeletal health [2,3], which may result in part through protective effects on mesenchymal stem cells. Prolonged skeletal unloading, such as during immobilization or space flight, leads to reduced bone mass and is also thought to negatively influence MSC function [4, 5]. Preservation of MSC function is therefore important for skeletal health and appears to be influenced by exercisegenerated signals.

Bone marrow serves as a primary repository for mesenchymal stem cells. MSC differentiation in bone marrow is primarily limited to the osteoblast and adipocyte lineages. Accrual of marrow fat is a hallmark of aging that may negatively influence stem cell function by promoting further adipogenesis from a limited precursor pool and through the production of deleterious cytokines [6]. Conditions that lead to adipocyte accumulation within the marrow, including immobilization and estrogen deficiency [7, 8], are associated with a reduction in osteoblast progenitors. A better understanding of environmental cues that help preserve MSC function and prevent adipogenesis within the marrow is needed.

Recent studies have shown that mechanical signals regulate MSC lineage allocation. Exercise-generated signals are pro-osteogenic and anti-adipogenic within the marrow cavity of rodents $[9,10]$, while hind limb unloading increases the potential for adipogenesis in ex vivo marrow cultures [11]. In vitro studies indicate that mechanical signals directly block adipogenic differentiation [12-14]. The ability of mechanical input to decrease adipogenesis is dependent on mechanical activation of $\beta$-catenin and is at least partially due to limiting expression of PPAR $\gamma 2[15,16]$, a primary adipogenic transcription factor. Whether mechanical factors can also interfere with adipogenesis through inhibition of PPAR $\gamma$ regulated transcription has not been determined.

PPAR $\gamma$ is a key transcription factor for adipocyte differentiation, with induction of target genes supporting emergence of a mature adipogenic phenotype [17]. PPAR $\gamma$ may also limit osteoblast differentiation through its ability to promote proteasomal degradation of $\beta$-catenin [18], a critical mediator of Wnt signaling. Importantly, haploinsufficiency of PPAR $\gamma$ is associated with reduced adipogenesis and augmented osteoblastogenesis [19], which suggests a primary role for PPAR $\gamma$ in MSC lineage allocation. Thus it is important to understand if mechanical factors restrict PPAR $\gamma$ actions, thereby enhancing the osteogenic potential of MSC.

In this work we evaluated the ability of mechanical signals to limit adipogenesis in mdMSC during PPAR $\gamma$ activation. Rosiglitazone, a thiazolidinedione PPAR $\gamma$ ligand, was used to induce adipogenesis. To enable analysis of single cell differentiation, we utilized a GFP reporter for the aP2 promoter, which is activated during adipogenesis and contains a target sequence for PPAR $\gamma$ binding [20]. Our results suggest that mechanical input restrains both PPAR $\gamma 2$ expression and action to preserve multipotentiality of the progenitor population. 


\section{Materials and methods}

\subsection{Reagents}

FBS was from Atlanta Biologicals (Atlanta, GA). Culture medium, trypsin-EDTA reagent, and antibiotics were purchased from Invitrogen (Carlsbad, CA). Insulin, indomethacin, and SB415286 were from Sigma-Aldrich (St. Louis, MO). PepMute Plus and LipoD293 transfection reagents were purchased from SignaGen Laboratories (Ijamsville, MD). Rosiglitazone was from Cayman Chemical (Ann Arbor, MI). BMP-2 was purchased from R\&D Systems (Minneapolis, MN).

\subsection{Cell culture}

mdMSC were generated from C57BL/6 wild-type mice using the procedure of Peister et al [21]. mdMSC were then plated at 3000 cells $/ \mathrm{cm}^{2}$ in Iscove's Modified Dulbecco's Medium containing $10 \% \mathrm{FBS}$ and $100 \mu \mathrm{g} / \mathrm{ml}$ penicillin/streptomycin for expansion from passage 5-10. For experiments, mdMSC were seeded at 5,000-10,000 cells $/ \mathrm{cm}^{2}$ in growth medium (a-MEM, 10\% FBS, antibiotics). Adipogenic medium consisted of $0.1 \mu \mathrm{M}$ dexamethasone, $5 \mu \mathrm{g} / \mathrm{ml}$ insulin, and $5 \mu \mathrm{M}$ rosiglitazone unless noted otherwise.

\subsection{Bicolor mdMSC}

C57BL/6 transgenic GFP reporter mice were intercrossed to produce the animals used to generate bicolor mdMSC. The SMaA-GFPmCherry mice, described previously [22], show strong expression in vascular smooth muscle and a weaker signal in the multipotential progenitor cells that are initially expressed in a bone marrow stromal cell culture or in rapidly regenerating bone. The aP2-GFPtopaz mouse line contains a $7.6 \mathrm{~kb}$ promoter fragment of the aP2/FABP4 gene that drives the pOB4 reporter cassette containing GFPtopaz [23]. The construct is expressed in actively forming brown and white fat in vivo as well as macrophages in the bone marrow and spleen. Female double positive F1 mice at 8 weeks of age were used to generate bicolor mdMSC following the same culture methods described above for the C57BL/6 wild-type mdMSC.

\subsection{Transient transfection with siRNA}

mdMSC were transfected with siRNA targeting $\beta$-catenin or a control nonsense siRNA at a concentration of $20 \mathrm{nM}$ using the PepMute Plus reagent in growth medium for $6 \mathrm{~h}$, followed by replacement with fresh growth medium. Experiments were initiated $24 \mathrm{~h}$ after transfection.

\subsection{Mechanical strain}

Concurrent with the addition of adipogenic supplements, a daily strain regimen ( $2 \%$ magnitude, $0.17 \mathrm{~Hz}, 3600$ cycles) was initiated using the Z-strain cell deformation device as previously described [12]. For strain experiments, mdMSC were plated on collagen-I coated silicone membranes.

\subsection{PPARy2 overexpression}

The FLAG-PPAR $\gamma 2$ plasmid was a gift from Dr. Monte Willis (UNC-Chapel Hill). mdMSC were transfected with pcDNA3.1(-)PPAR $\gamma 2$ or pcDNA3.1 expression vector (6 well plates at $0.8 \mu \mathrm{g}$ DNA/well; strain dishes at $5 \mu \mathrm{g}$ DNA/dish) using LipoD293 reagent $(3 \mu \mathrm{l} / \mu \mathrm{g}$ DNA). Twenty-four hours after transfection, medium was changed to adipogenic medium. 


\section{7. aP2 luciferase assay}

Cells were co-transfected with FLAG-PPAR $\gamma 2(0.5 \mu \mathrm{g})$ and pCMV-RXRa $(0.5 \mu \mathrm{g})$ as well as with the aP2-luciferase construct [20] $(0.5 \mu \mathrm{g})$ versus salmon sperm DNA. The pCMVRXRa plasmid [24] was a gift from Dr. Mark Nanes (Emory University). The aP2luciferase construct was from Addgene (plasmid 8858, deposited by Bruce Spiegelman). $\beta$ galactosidase $(0.2 \mu \mathrm{g})$ was used to control for transfection efficiency in all wells. LipoD293 reagent was used according to the manufacturer's protocol. The medium was changed to $a-$ $\mathrm{MEM} \pm 1 \mu \mathrm{M}$ rosiglitazone $24 \mathrm{~h}$ after transfection and treatments applied. Luciferase assays were performed on cell lysates collected $3 \mathrm{~d}$ after transfection with the luciferase assay system (Promega) and Galacto-Star (Applied Biosystems, Bedford, MA) and measured using the GloMax Luminometer (Promega).

\subsection{Western blotting}

Whole cell lysates were prepared as previously described $[16,25]$ and protein $(10-35 \mu \mathrm{g})$ was separated on a polyacrylamide gel before transfer to PVDF membrane. Antibodies directed against $\beta$-catenin (BD Biosciences), aP2 (ProSci, Inc., Poway, CA), active $\beta$ catenin, PPAR $\gamma$, adiponectin, and $\beta$-tubulin (Santa Cruz Biotechnology, Santa Cruz, CA) were used. The antibody for active $\beta$-catenin was specific for the hypo-phosphorylated form of $\beta$-catenin [26]. Secondary antibody conjugated with horseradish peroxidase was detected by chemiluminescence. Images were acquired with a Hewlett-Packard Scanjet and densitometry determined using NIH ImageJ, $1.37 \mathrm{v}$.

\subsection{Histochemistry}

Cultures fixed in $10 \%$ formalin were stained with $0.5 \%$ oil red O (Poly Scientific, Bay Shore, NY) to detect cytoplasmic triglyceride droplets.

\subsection{Flow cytometry}

Bicolor mdMSC were cultured in specified medium for 1-4 d. For flow analysis, single cell suspensions were prepared by treating cultures with trypsin-EDTA for 5 min to release cells from the culture dish. Cells from multiple wells were pooled into a single sample and centrifuged. The cell pellet was re-suspended in PBS containing 1\% paraformaldehyde and stored at $4^{\circ} \mathrm{C}$ until flow analysis was performed using a BD LSRII cytometer. mdMSC from wild-type C57BL/6 mice were used as an unstained, negative control for flow analysis. Positive GFP expression was defined as the intensity corresponding to $0.1 \%$ positive cells in the negative control. For cultures evaluated by fluorescent microscopy (IX81, Nikon), PBS containing $2 \%$ paraformaldehyde was added to wells and plates were stored at $4^{\circ} \mathrm{C}$ until microscopy was performed. For sorting of bicolor mdMSC, cells were cultured in specified medium in $100 \mathrm{~mm}$ diameter dishes. Single cell suspensions were prepared as described above and cells from multiple dishes were pooled into a single sample. Sorting of bicolor mdMSC by GFP intensity was performed under sterile conditions using a Dako MoFlo sorter at the UNC Flow Cytometry Core Facility. Sorted mdMSC were replated $(15,000$ cells $/ \mathrm{cm}^{2}$ ) for further culture.

\subsection{Real-time RT-PCR}

Total RNA was isolated and reverse transcribed as in [25]. Aliquots of cDNA were diluted 5-5000 fold to generate relative standard curves. Osterix and 18S primers were as previously described [16]. PCR products were normalized to $18 \mathrm{~S}$ amplicons in the RT sample.

\subsection{Statistical analysis}

Results are expressed as the mean \pm SEM. Statistical significance was evaluated by $t$-test, one-way ANOVA with a Tukey's post hoc test, or two-way ANOVA with a Bonferroni 
posttest (GraphPad Prism 5.04) where appropriate. All experiments were replicated at least once. Densitometry data were compiled from three separate experiments.

\section{Results}

\subsection{GSK3 $\beta$ inhibition disrupts rosiglitazone-directed adipogenesis through $\boldsymbol{\beta}$-catenin}

To study potential mechanical effects on PPAR $\gamma$ transcriptional activity and regulation of its target genes, we used the thiazolidinedione PPAR $\gamma$ ligand rosiglitazone to induce adipogenesis in marrow derived MSC. Rosiglitazone in combination with insulin and dexamethasone rapidly stimulated adipogenic differentiation as evidenced by expression of adipogenic proteins (Fig. 1A). mdMSC expressed PPAR $\gamma 1$ but not PPAR $\gamma 2$ in the absence of adipogenic stimuli (left lane). Treatment with rosiglitazone increased expression of both PPAR $\gamma 1$ and PPAR $\gamma 2$. aP2 and adiponectin, gene targets of PPAR $\gamma 2$, were detected by immunoblot in day 2 cultures and protein levels were further increased on day 4 . The levels of active and total $\beta$-catenin were reduced in day 4 cultures in response to rosiglitazone treatment compared to untreated cultures (left lane).

Cellular $\beta$-catenin accumulation stimulated via canonical Wnt signaling or pharmacologic inhibition of GSK3 $\beta$ disrupts adipogenesis and reduces expression of PPAR $\gamma$ [16]. We here asked whether inhibition of GSK $3 \beta$ could limit rosiglitazone-induced adipogenesis in mdMSC, e.g., at targets distal to PPAR $\gamma$ activation. Rosiglitazone induction of PPARy2, $\mathrm{aP} 2$, and adiponectin proteins was blocked by treatment with the GSK3 $\beta$ inhibitor SB415286 (Fig. 1B). Concomitantly, SB415286 also limited accumulation of lipid droplets (Fig. 1C). The ratio of active $\beta$-catenin/tubulin was increased $60 \%$ with SB415286 treatment, from a ratio of 0.9 in cultures without SB415286 to a ratio of 1.4 in cultures

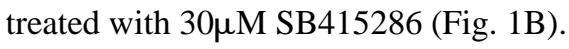

As GSK3 $\beta$ inhibition affects multiple targets, we queried whether the increase in $\beta$-catenin was responsible for restraining rosiglitazone-directed adipogenesis. The blot for active $\beta$ catenin showed near complete knockdown by siRNA targeting $\beta$-catenin (Fig. 1D). In cultures where $\beta$-catenin was silenced, SB415286 treatment did not block expression of the adipogenic proteins PPARy2, aP2, and adiponectin stimulated by rosiglitazone. Thus, GSK $3 \beta$ inhibition restricts PPAR $\gamma 2$ expression and disrupts rosiglitazone-induced adipogenesis through $\beta$-catenin.

\subsection{Mechanical strain inhibits adipogenesis induced by rosiglitazone}

We next evaluated the ability of mechanical signals to limit adipogenesis induced by rosiglitazone. Daily treatment with mechanical strain $(2 \%, 0.17 \mathrm{~Hz}, 3600$ cycles) applied for 3 consecutive days to mdMSC during rosiglitazone treatment inhibited adipogenic differentiation. Shown in Fig. 2A, strain reduced induction of PPAR $\gamma 2$ as well as the expression of its downstream targets, aP2 and adiponectin, in the presence of rosiglitazone treatments $(1-25 \mu \mathrm{M})$. The effect of strain to limit rosiglitazone-induced accumulation of adipogenic proteins was verified by densitometry performed over a series of 3 experiments (Fig. 2B). Compared to the control unstrained cultures, strain significantly reduced expression of PPAR $\gamma 2, a \mathrm{P} 2$, and adiponectin by $88 \pm 3 \%, 64 \pm 5 \%$, and $77 \pm 4 \%$, respectively.

To investigate the role of $\beta$-catenin in mediating strain inhibition of rosiglitazone-directed adipogenesis, $\beta$-catenin was silenced. In cultures where the level of active $\beta$-catenin protein was reduced by more than $80 \%$ by siRNA treatment, adiponectin protein expression was not inhibited by application of strain (Fig. 2C). In contrast, strain inhibition of PPAR $\gamma 2$ persisted in one of two experiments where $\beta$-catenin was silenced. This suggests that strain has effects on PPAR $\gamma 2$ activity as well as PPAR $\gamma 2$ expression, which we address in the 
next section, and indicates a primary role for $\beta$-catenin in strain repression of rosiglitazone's induction of adiponectin.

\subsection{Mechanical strain interferes with PPARy action}

As discussed above, treatment with either a daily mechanical regimen or with the GSK3 $\beta$ inhibitor SB415286 limited induction of PPAR $\gamma 2$ expression by rosiglitazone. The repression of aP2 and adiponectin by these treatments could be ascribed to either the reduction in PPAR $\gamma 2$ expression or to a repression of PPAR $\gamma$ activity. To delineate an effect of $\beta$-catenin distal to PPAR $\gamma 2$ expression, we set out to determine whether adipogenesis induced by rosiglitazone could by inhibited in MdMSC where PPAR $\gamma 2$ was overexpressed. A PPAR $\gamma 2$-FLAG vector was transfected into mdMSC, and adipogenic medium containing rosiglitazone was added to cultures 24 hours after transfection. By 4 days post-transfection, PPAR $\gamma 2$ protein was strongly expressed in mdMSC transfected with the overexpression vector (Fig. 3A). GSK3 $\beta$ inhibition effectively blocked adipogenesis in mdMSC despite abundant PPAR $\gamma 2$ expression, indicated by limited expression of aP2 and adiponectin proteins in SB415286 treated cultures overexpressing PPAR $\gamma 2$. These data indicate that $\beta$-catenin interferes with induction of PPAR $\gamma 2$ target genes.

A luciferase construct driven by the aP2 promoter [20] was used to evaluate the effect of GSK $3 \beta$ inhibition on PPAR $\gamma$ transactivation. In cells overexpressing PPAR $\gamma 2$-FLAG and RXRa SB415286 treatment decreased aP2 transcription by $50 \pm 2 \%$ in basal medium without rosiglitazone (Fig. 3B). The addition of rosiglitazone increased PPAR $\gamma 2 / \mathrm{RXRa}$ induced transcription of aP2 by 2 -fold. SB415286 treatment significantly decreased aP2 transcription by $34 \pm 6 \%$ even in the presence of rosiglitazone. Thus, notwithstanding high levels of PPAR $\gamma 2$ and an activating ligand, GSK3 $\beta$ inhibition reduced transcriptional activity of PPAR $\gamma 2$, suggesting that $\beta$-catenin does limit PPAR $\gamma 2$ transactivation in mdMSC.

Mechanical strain also effectively limited rosiglitazone-induced adipogenesis in mdMSC overexpressing PPAR $\gamma 2$ (Fig. 3C). The daily strain regimen reduced expression of both aP2 and adiponectin, although the level of transfected PPAR $\gamma 2$ was unaffected by strain. Next, the aP2-luciferase construct was used to directly assess strain effects on PPAR $\gamma 2$ transactivation. mdMSC overexpressing PPAR $\gamma 2 / \mathrm{RXRa}$ were treated with rosiglitazone. When mechanical strain was applied concurrently with rosiglitazone, PPAR $\gamma 2 / \mathrm{RXRa}$ induced transcription of aP2 was significantly reduced by $18 \pm 3 \%$ (Fig. 3D). These experiments indicate that strain inhibition of adipogenesis involves limiting ligand-activated PPAR $\gamma 2$ target gene transactivation.

\subsection{Evaluating adipogenesis in individual cells using an aP2-GFP reporter}

Although measuring whole culture expression of adipogenic proteins within a population of differentiating mdMSC showed that expression of an adipocyte phenotype induced by rosiglitazone was constrained by mechanical input, this method of analysis did not allow us to determine where the mechanical block occurred. Limitation of adipogenesis could be through reducing allocation of undifferentiated cells to the adipocyte lineage, or through limiting PPAR $\gamma$ action within cells which had already entered the adipocyte lineage. Adipogenic cultures exposed to a daily strain regimen were found to have an enhanced potential to enter the osteogenic lineage compared to control adipogenic cultures [16], suggesting mechanical disruption of adipogenic commitment. To allow analysis at the level of single cells, we generated a mdMSC population from adult transgenic mice whose cells contained a GFP reporter driven by a $7.6 \mathrm{kB}$ promoter fragment of the aP2 gene to serve as an indicator of entry into the adipogenic lineage [23]. The mice also possessed a mCherry reporter that was positively regulated by SMaA and therefore expressed in MSC [22]. 
In the absence of adipogenic supplements (i.e. basal medium), the majority of bicolor mdMSC $(84.1 \%)$ were positive for SMaA-mCherry, but negative for aP2-GFP after 3 days in culture. The total fraction of cells positive for GFP in the basal medium was $8.8 \%$ (Fig. 4A). The total fraction of GFP-positive cells increased to $34.3 \%$ after 3 days of treatment with insulin and dexamethasone, a combination shown to induce a gradual adipogenic differentiation over 7-10 days in mdMSC [13]. The addition of rosiglitazone greatly accelerated adipogenesis: GFP accumulation was seen in $89.8 \%$ of cells after 3 days in culture and GFP expression was confirmed by microscopy (Fig. 4B). In the presence of adipogenic supplements, the majority of GFP-positive cells were also positive for SMaAmCherry.

In the presence of rosiglitazone, activation of the aP2-GFP construct was rapid. A detectable level of GFP was measured in 59\% of cells after only 1 day of treatment (Fig. 4C). This early aP2 response may be due to activation of PPAR $\gamma 1$ [27], which is constitutively expressed in mdMSC (Fig. 4D). Shown in 4C, the fraction of cells positive for GFP continued to increase from day 1 to 4 along with a significant increase in GFP median fluorescence. Thus, accrual of GFP protein increased along with expression of PPAR $\gamma 2$ and other adipogenic proteins (Fig. 4D).

These findings indicate that aP2-GFP reporter intensity reflects progression along the adipogenic lineage. mdMSC treated with rosiglitazone, insulin, and dexamethasone for 3 days were sorted into two subpopulations based upon GFP intensity. The subpopulation with the highest GFP intensity (top quintile of total cell population; defined as the upper fraction or "UF") was separated from the remainder of the cell population (defined as the lower fraction or "LF"). Comparing the adipogenic proteins present in the sorted cell populations revealed that the upper fraction contained more PPAR $\gamma 2$, aP2, and adiponectin proteins compared to the lower fraction (Fig. 5A). This data indicates that the upper fraction of GFPexpressing cells represented a more committed adipogenic population compared to the remainder of GFP-expressing cells.

To further characterize the two cell subpopulations defined by GFP intensity, we asked whether there was a difference in their multipotentiality. After 3 days in adipogenic medium containing rosiglitazone, mdMSC were sorted by GFP intensity into the upper and lower fractions (UF; LF). Following sorting, each fraction was re-plated for further culture. To assess multipotentiality, the sorted fractions were treated with BMP-2 for 2 days at a dose known to induce osteogenesis [16]. In the absence of BMP-2, there was minimal expression of the osteoblast transcription factor osterix in either fraction (Fig. 5B). Treatment with BMP-2 induced expression of osterix by 42.3-fold in the lower fraction of rosiglitazonetreated cultures. Although BMP-2 did increase osterix expression in the upper fraction by 11.6-fold, the increase in osterix was significantly less than that in the lower fraction. In sum, adipogenic mdMSC expressing high levels of aP2-GFP were resistant to an osteogenic stimulus, reflecting a population of mdMSC terminally committed to an adipocyte lineage.

\subsection{Strain restricts appearance of highly adipogenic populations}

We next used mdMSC containing the aP2-GFP construct to evaluate how mechanical strain affected the proportion of cells with high and low GFP expression by addressing the following question: would mechanical input reduce global aP2-GFP expression, or specifically limit the proportion of cells in the upper fraction enriched with committed adipocytes? First, we demonstrated that daily application of strain blocked adipogenesis induced by rosiglitazone in the mdMSC containing the aP2-GFP construct. Expression of PPAR $\gamma 2, a P 2$, and adiponectin proteins was strongly decreased in strained cultures (Fig. 6A), and confirmed by densitometry (Fig. 6B). As such, the ability of mechanical input to 
limit expression of adipocyte proteins in mdMSC containing the aP2-GFP construct was similar to the strain effect in wild-type mdMSC (see Fig. 2A).

We next evaluated strain effects on aP2-GFP expression. Mechanical strain did not significantly reduce the total number of GFP positive cells induced by rosiglitazone (Fig. 6C) suggesting that the initial aP2-GFP response to rosiglitazone was largely unaffected. However, the median intensity of GFP expression was decreased by $31 \pm 7 \%$ in the strained cultures, an effect that approached significance $(\mathrm{p}=0.052)$. This result suggested that strain might preferentially block the maturation of mdMSC into committed adipocytes. Guided by the GFP subpopulation analysis as detailed in figure 5, the GFP intensity threshold that identified the top quintile of GFP-expressing cells in mdMSC cultures was applied to flow analysis of the strained cultures to measure how strain might alter this highly adipogenic population. The portion of GFP-expressing cells above this threshold was $10.0 \pm 1.2 \%$ in strained cultures, a significant two-fold reduction from the portion of cells above the threshold in unstrained cultures $(20.1 \pm 0.1 \%)$. Together with the effect of strain to almost completely block appearance of adipogenic proteins (Fig. 6A), this analysis points to an effect of mechanical input to prevent late commitment to the adipogenic lineage. Importantly, as we have shown that the upper fraction of aP2-GFP expressing cells has a reduced ability to respond to osteogenic cues, the ability of strain to specifically limit the size of this highly adipogenic population indicates that the multipotentiality of the mdMSC population is preserved by mechanical strain.

To further test the thesis that mechanical input preserves multipotentiality by limiting commitment to an adipogenic lineage, we evaluated strain effects on adipogenesis in mdMSC containing the aP2-GFP construct under conditions where adipogenesis was stimulated in the absence of an exogenous PPAR $\gamma$ ligand. To this end we used the cyclooxygenase inhibitor indomethacin, which is a strong enhancer of adipogenesis when added to medium containing dexamethasone and insulin [28]. When mdMSC containing the aP2-GFP construct were treated with indomethacin $(50 \mu \mathrm{M})$ in combination with insulin and dexamethasone, adipogenic differentiation was induced, as indicated by expression of PPAR $\gamma 2$, aP2, and adiponectin proteins (Fig. 7A). In comparison to indomethacin, the effect of rosiglitazone $(5 \mu \mathrm{M})$ was more rapid and induced greater expression of adipogenic proteins during the first 4 days of culture. GFP expression was detected in $61.1 \%$ of cells after 3 days of treatment with indomethacin (Fig. 7B) and was confirmed by microscopy (Fig. 7C).

FACs sorting was used to divide the cell population into an upper and lower fraction based upon aP2-GFP intensity as described in Figure 5. Immunoblots of whole cell lysates prepared from the sorted cell populations revealed that the upper fraction contained more PPAR $\gamma 2, a P 2$, and adiponectin proteins compared to the lower fraction (Fig. 7D), similar to the sorted populations analyzed in rosiglitazone-treated cultures. When the two sorted populations were re-plated and exposed to BMP-2 for two days, the upper fraction of indomethacin-treated cultures was resistant to osteogenic induction by BMP-2 (Fig. 7E). Accordingly, BMP-2 increased osterix expression by 44.6-fold in the lower fraction but only 16.6-fold in the upper fraction, consistent with adipocyte commitment in the upper fraction.

Application of a daily strain regimen significantly reduced the total number of GFP positive cells induced by indomethacin by $20 \%$, from $84.5 \pm 2.8 \%$ in unstrained cultures to $67.6 \pm$ $1.0 \%$ in strained cultures after 4 days of treatment (Fig. 7F). To further evaluate strain effects on the committed adipocyte population, the GFP intensity threshold which identified the top quintile of GFP-expressing cells in mdMSC cultures was applied to flow analysis of the strained cultures. The portion of GFP-expressing cells above this threshold was $7.3 \pm$ $1.2 \%$ in strained cultures, a significant reduction of more than half from the portion of cells 
above the threshold in unstrained cultures $(20.2 \pm 0.2 \%)$. Consistent with strain effects on aP2-GFP expression, mechanical strain inhibited expression of adipogenic proteins in mdMSC stimulated with indomethacin (Fig. 7G). Thus, strain appeared to preferentially disrupt late maturation of mdMSC into committed adipocytes induced by indomethacin. In sum, these data indicate that mechanical treatment preserves multipotentiality of mdMSC in the face of adipogenic stimuli.

\section{Discussion}

Conditions that promote skeletal fragility, including aging and forced unloading, are associated with progressive accumulation of adipocytes within the bone marrow. Stem cell function within the marrow may be negatively impacted by the presence of adipocytes. Not only does exercise increase bone quantity and reduce skeletal fragility, but it can also reduce bone marrow adipogenesis. Mechanical factors have direct effects on mesenchymal stem cells, preventing adipocyte differentiation. In the work presented here, we have shown that mechanical input blocks adipogenesis and preserves multipotentiality of marrow-derived MSC under conditions promoting PPAR $\gamma$ activation. Thus, mechanical factors generated by weight-bearing exercise are potent environmental cues that protect MSC function by limiting differentiation under strongly adipogenic conditions, thereby benefiting overall skeletal health.

In this work, the PPAR $\gamma$ ligand rosiglitazone was used to induce adipogenesis to study mechanical effects on PPAR $\gamma 2$ regulation of target genes. Rosiglitazone treatment led to the induction of PPAR $\gamma 2$ expression in murine marrow-derived MSC, supporting rapid adipocyte differentiation, consistent with prior studies evaluating rosiglitazone effects on MSC [29, 30]. Application of a daily strain regimen inhibited rosiglitazone-induced expression of PPAR $\gamma 2$, as well as expression of its target genes aP2 and adiponectin, indicating that mechanical input exerts a primary effect to limit PPAR $\gamma$ induction. However, mechanical strain also blocked expression of aP2 and adiponectin in the presence of PPAR $\gamma 2$ overexpression, indicating that inhibition of PPAR $\gamma 2$ action contributes to mechanical repression of adipogenesis.

Using mdMSC containing an aP2 directed GFP allowed separation by GFP intensity of a population which had high expression of PPAR $\gamma 2$ and adiponectin; immunoblotting indicated that the majority of expression of adipogenic proteins derived from the subpopulation (top quintile) with the highest GFP intensity. Importantly, this most terminally differentiated population was significantly less responsive to an osteogenic stimulus. Application of strain to aP2-GFP expressing cells reduced the number of cells expressing the highest GFP intensity, indicative of the terminally differentiated phenotype, by at least half, an effect consistent with the reduction in adiponectin and aP2 proteins. Taken together, this data indicates that mechanical input preserves MSC, in the face of strong adipogenic stimuli, in a multipotential state. Preservation of multipotentiality is critical for bone health. Senescent osteoporosis can be attributed, in part, to a reduction in MSC potential [31].

Marrow-derived MSC containing the aP2-GFP reporter responded rapidly to treatment with a PPAR $\gamma$ ligand in the MSC population harvested from adult animals used in this study. The protocol developed by Peister [21] generates a multipotent MSC population which undergoes rapid adipogenic differentiation [15], markedly faster than adipogenesis observed in primary cultures [32]. The isolation and expansion protocol may have influenced the composition of the bicolor mdMSC population used in our study, possibly allowing for a shift in the mdMSC pool to favor a subpopulation that has enhanced adipogenic potential. However, despite the fact that the majority of bicolor mdMSC expressed aP2-GFP in 
response to rosiglitazone, a much smaller percentage of these cells went on to become terminally differentiated adipocytes during this time period.

A role for $\beta$-catenin in the mechanical repression of PPAR $\gamma 2$ expression has been demonstrated for a variety of adipogenic stimuli $[12,13,15]$. In our work, although $\beta$ catenin was not found to have a consistent role in strain inhibition of PPAR $\gamma 2$ expression induced by rosiglitazone treatment, this pathway was critically involved in the strain effect on adiponectin. In the case of rosiglitazone induced adipogenesis, mechanically-activated $\beta$ catenin participates in suppression of PPAR $\gamma$ actions. $\beta$-catenin has been shown to limit PPAR $\gamma$ regulated transcription in early adipocytes $[18,33]$, suggesting a negative interaction between $\beta$-catenin and PPAR $\gamma 2$ itself. This idea is supported by our data showing that sustained GSK3 $\beta$ inhibition by SB415286, leading to $\beta$-catenin accumulation, decreased aP2-luciferase activity and greatly reduced expression of aP2 and adiponectin in mdMSC overexpressing PPAR $\gamma 2$.

Our laboratory has defined aspects of mechanical signaling that result in $\beta$-catenin preservation and activation in MSC. Similar to Wnt stimulated $\beta$-catenin activation, a release from tonic GSK3 $\beta$ phosphorylation via mechanical input prevents $\beta$-catenin processing by proteosomes. However, in contradistinction to the canonical Wnt signaling pathway where GSK3 $\beta$ is sequestered away from $\beta$-catenin downstream of the Wnt coreceptors Lrp and frizzled [34], mechanical stimulation requires activation of mTORC2 [35], a complex containing mTOR and rictor that is insensitive to rapamycin inhibition [36]. The target of mTORC2 activation by strain is Akt, which phosphorylates and inactivates GSK3 $\beta$ $[35,37]$, leading to $\beta$-catenin activation. Importantly, a recent in vivo study showed that exercise increases GSK3 $\beta$ phosphorylation, resulting in increased $\beta$-catenin and decreased PPAR $\gamma$ measured in lumbar vertebrae of ovariectomized rats [38].

In sum, we have shown that mechanical strain inhibits rosiglitazone-induced adipogenesis in marrow-derived MSC, a process that involves limiting PPAR $\gamma 2$ expression. Strain can also regulate events downstream of PPAR $\gamma$ activation, as expression of $\mathrm{aP} 2$ and adiponectin is blocked by strain application in mdMSC overexpressing PPAR $\gamma 2$ treated with rosiglitazone. Furthermore, we have used mdMSC containing an aP2-GFP construct to demonstrate that strain preferentially restricts maturation of progenitor cells into committed adipocytes, preserving multipotentiality of the mdMSC population. As such, mechanically stimulated MSC are more capable of responding to osteogenic factors. Our data suggest that exercise should be a useful countermeasure to conditions that promote adipogenesis within bone marrow, including aging, immobility, and pharmacologic treatments that activate PPAR $\gamma$.

\section{Acknowledgments}

This work was supported by National Institutes of Health grants AR42360 and AR56655 to JR and the UNC BIRCWH career development award 5K12HD001441-12 to MS. The UNC Flow Cytometry Core Facility is supported in part by an NCI Center Core Support Grant (P30CA06086) to the UNC Lineberger Comprehensive Cancer Center.

\section{References}

1. Zaim M, Karaman S, Cetin G, Isik S. Donor age and long-term culture affect differentiation and proliferation of human bone marrow mesenchymal stem cells. Ann Hematol. 2012

2. Guadalupe-Grau A, Fuentes T, Guerra B, Calbet JA. Exercise and bone mass in adults. Sports Med. 2009; 39:439-68. [PubMed: 19453205]

3. Gomez-Cabello A, Ara I, Gonzalez-Aguero A, Casajus JA, Vicente-Rodriguez G. Effects of training on bone mass in older adults: a systematic review. Sports Med. 2012; 42:301-25. [PubMed: 22376192] 
4. Wronski TJ, Morey-Holton E, Jee WS. Skeletal alterations in rats during space flight. Adv Space Res. 1981; 1:135-40. [PubMed: 11541702]

5. Zayzafoon M, Gathings WE, McDonald JM. Modeled microgravity inhibits osteogenic differentiation of human mesenchymal stem cells and increases adipogenesis. Endocrinology. 2004; 145:2421-32. [PubMed: 14749352]

6. Ouchi N, Parker JL, Lugus JJ, Walsh K. Adipokines in inflammation and metabolic disease. Nat Rev Immunol. 2011; 11:85-97. [PubMed: 21252989]

7. Yeung DK, Griffith JF, Antonio GE, Lee FK, Woo J, Leung PC. Osteoporosis is associated with increased marrow fat content and decreased marrow fat unsaturation: a proton MR spectroscopy study. J Magn Reson Imaging. 2005; 22:279-85. [PubMed: 16028245]

8. Minaire P, Edouard C, Arlot M, Meunier PJ. Marrow changes in paraplegic patients. Calcif Tissue Int. 1984; 36:338-40. [PubMed: 6432298]

9. David V, Martin A, Lafage-Proust MH, Malaval L, Peyroche S, Jones DB, Vico L, Guignandon A. Mechanical loading down-regulates peroxisome proliferator-activated receptor gamma in bone marrow stromal cells and favors osteoblastogenesis at the expense of adipogenesis. Endocrinology. 2007; 148:2553-62. [PubMed: 17317771]

10. Menuki K, Mori T, Sakai A, Sakuma M, Okimoto N, Shimizu Y, Kunugita N, Nakamura T. Climbing exercise enhances osteoblast differentiation and inhibits adipogenic differentiation with high expression of PTH/PTHrP receptor in bone marrow cells. Bone. 2008; 43:613-20. [PubMed: 18567552]

11. Pan Z, Yang J, Guo C, Shi D, Shen D, Zheng Q, Chen R, Xu Y, Xi Y, Wang J. Effects of hindlimb unloading on ex vivo growth and osteogenic/adipogenic potentials of bone marrow-derived mesenchymal stem cells in rats. Stem Cells Dev. 2008; 17:795-804. [PubMed: 18710346]

12. Sen B, Styner M, Xie Z, Case N, Rubin CT, Rubin J. Mechanical loading regulates NFATC1 and \{beta\}-catenin signaling through a GSK3 $\{$ beta $\}$ control node. J Biol Chem. 2009

13. Sen B, Xie Z, Case N, Styner M, Rubin CT, Rubin J. Mechanical signal influence on mesenchymal stem cell fate is enhanced by incorporation of refractory periods into the loading regimen. $\mathrm{J}$ Biomech. 2011; 44:593-9. [PubMed: 21130997]

14. Yang X, Cai X, Wang J, Tang H, Yuan Q, Gong P, Lin Y. Mechanical stretch inhibits adipogenesis and stimulates osteogenesis of adipose stem cells. Cell Prolif. 2012; 45:158-66. [PubMed: 22229452]

15. Case N, Xie Z, Sen B, Styner M, Zou M, O’Conor C, Horowitz M, Rubin J. Mechanical activation of beta-catenin regulates phenotype in adult murine marrow-derived mesenchymal stem cells. J Orthop Res. 2010; 28:1531-8. [PubMed: 20872592]

16. Sen B, Xie Z, Case N, Ma M, Rubin C, Rubin J. Mechanical strain inhibits adipogenesis in mesenchymal stem cells by stimulating a durable beta-catenin signal. Endocrinology. 2008; 149:6065-75. [PubMed: 18687779]

17. Farmer SR. Regulation of PPARgamma activity during adipogenesis. Int J Obes (Lond). 2005; 29(Suppl 1):S13-6. [PubMed: 15711576]

18. Liu J, Wang H, Zuo Y, Farmer SR. Functional interaction between peroxisome proliferatoractivated receptor gamma and beta-catenin. Mol Cell Biol. 2006; 26:5827-37. [PubMed: 16847334]

19. Akune T, Ohba S, Kamekura S, Yamaguchi M, Chung UI, Kubota N, Terauchi Y, Harada Y, Azuma Y, Nakamura K, Kadowaki T, Kawaguchi H. PPARgamma insufficiency enhances osteogenesis through osteoblast formation from bone marrow progenitors. J Clin Invest. 2004; 113:846-55. [PubMed: 15067317]

20. Graves RA, Tontonoz P, Spiegelman BM. Analysis of a tissue-specific enhancer: ARF6 regulates adipogenic gene expression. Mol Cell Biol. 1992; 12:1202-8. [PubMed: 1545801]

21. Peister A, Mellad JA, Larson BL, Hall BM, Gibson LF, Prockop DJ. Adult stem cells from bone marrow (MSCs) isolated from different strains of inbred mice vary in surface epitopes, rates of proliferation, and differentiation potential. Blood. 2004; 103:1662-8. [PubMed: 14592819]

22. Kalajzic Z, Li H, Wang LP, Jiang X, Lamothe K, Adams DJ, Aguila HL, Rowe DW, Kalajzic I. Use of an alpha-smooth muscle actin GFP reporter to identify an osteoprogenitor population. Bone. 2008; 43:501-10. [PubMed: 18571490] 
23. Kronenberg K, Harrison J, Rowe DW. Mapping the Interrelationship of the Adipogenic and Osteogenic Lineage with Visual Reporters. Journal of Bone and Mineral Research. 2008; 23 Abstract M097.

24. Farmer PK, He X, Schmitz ML, Rubin J, Nanes MS. Inhibitory effect of NF-kappaB on 1,25dihydroxyvitamin $\mathrm{D}(3)$ and retinoid $X$ receptor function. Am J Physiol Endocrinol Metab. 2000; 279:E213-20. [PubMed: 10893342]

25. Case N, Ma M, Sen B, Xie Z, Gross TS, Rubin J. Beta-catenin levels influence rapid mechanical responses in osteoblasts. J Biol Chem. 2008; 283:29196-205. [PubMed: 18723514]

26. van Noort M, Meeldijk J, van der Zee R, Destree O, Clevers H. Wnt signaling controls the phosphorylation status of beta-catenin. J Biol Chem. 2002; 277:17901-5. [PubMed: 11834740]

27. Lehmann JM, Moore LB, Smith-Oliver TA, Wilkison WO, Willson TM, Kliewer SA. An antidiabetic thiazolidinedione is a high affinity ligand for peroxisome proliferator-activated receptor gamma (PPAR gamma). J Biol Chem. 1995; 270:12953-6. [PubMed: 7768881]

28. Styner M, Sen B, Xie Z, Case N, Rubin J. Indomethacin promotes adipogenesis of mesenchymal stem cells through a cyclooxygenase independent mechanism. J Cell Biochem. 2010; 111:104250. [PubMed: 20672310]

29. Lecka-Czernik B, Gubrij I, Moerman EJ, Kajkenova O, Lipschitz DA, Manolagas SC, Jilka RL. Inhibition of Osf2/Cbfa1 expression and terminal osteoblast differentiation by PPARgamma2. J Cell Biochem. 1999; 74:357-71. [PubMed: 10412038]

30. Ninomiya Y, Sugahara-Yamashita Y, Nakachi Y, Tokuzawa Y, Okazaki Y, Nishiyama M. Development of a rapid culture method to induce adipocyte differentiation of human bone marrow-derived mesenchymal stem cells. Biochem Biophys Res Commun. 2010; 394:303-8. [PubMed: 20206132]

31. Bonyadi M, Waldman SD, Liu D, Aubin JE, Grynpas MD, Stanford WL. Mesenchymal progenitor self-renewal deficiency leads to age-dependent osteoporosis in Sca-1/Ly-6A null mice. Proc Natl Acad Sci U S A. 2003; 100:5840-5. [PubMed: 12732718]

32. Lecka-Czernik B, Moerman EJ, Grant DF, Lehmann JM, Manolagas SC, Jilka RL. Divergent effects of selective peroxisome proliferator-activated receptor-gamma 2 ligands on adipocyte versus osteoblast differentiation. Endocrinology. 2002; 143:2376-84. [PubMed: 12021203]

33. Liu J, Farmer SR. Regulating the balance between peroxisome proliferator-activated receptor gamma and beta-catenin signaling during adipogenesis. A glycogen synthase kinase 3 beta phosphorylation-defective mutant of beta-catenin inhibits expression of a subset of adipogenic genes. J Biol Chem. 2004; 279:45020-7. [PubMed: 15308623]

34. Taelman VF, Dobrowolski R, Plouhinec JL, Fuentealba LC, Vorwald PP, Gumper I, Sabatini DD, De Robertis EM. Wnt signaling requires sequestration of glycogen synthase kinase 3 inside multivesicular endosomes. Cell. 2010; 143:1136-48. [PubMed: 21183076]

35. Case N, Thomas J, Sen B, Styner M, Xie Z, Galior K, Rubin J. Mechanical regulation of glycogen synthase kinase 3beta (GSK3beta) in mesenchymal stem cells is dependent on Akt protein serine 473 phosphorylation via mTORC2 protein. J Biol Chem. 2011; 286:39450-6. [PubMed: 21956113]

36. Foster KG, Fingar DC. Mammalian target of rapamycin (mTOR): conducting the cellular signaling symphony. J Biol Chem. 2010; 285:14071-7. [PubMed: 20231296]

37. Sen B, Guilluy C, Xie Z, Case N, Styner M, Thomas J, Oguz I, Rubin C, Burridge K, Rubin J. Mechanically induced focal adhesion assembly amplifies anti-adipogenic pathways in mesenchymal stem cells. Stem Cells. 2011; 29:1829-36. [PubMed: 21898699]

38. Bu S, Chen Y, Wang S, Zhang F, Ji G. Treadmill training regulates beta-catenin signaling through phosphorylation of GSK-3beta in lumbar vertebrae of ovariectomized rats. Eur J Appl Physiol. 2012

\section{abbreviations}

mdMSC marrow-derived mesenchymal stem cells

FBS fetal bovine serum 
SMaA

smooth muscle alpha-actin 


\section{Highlights}

- Mechanical strain inhibits rosiglitazone-induced adipogenesis in marrowderived MSC

- Strain repression of adipogenesis involves limiting PPAR $\gamma 2$ expression

- Mechanical strain blocks expression of aP2 and adiponectin in mdMSC overexpressing PPAR $\gamma 2$ treated with rosiglitazone

- mdMSC containing an aP2-GFP construct were used to demonstrate that strain preferentially restricts maturation of progenitor cells into committed adipocytes 


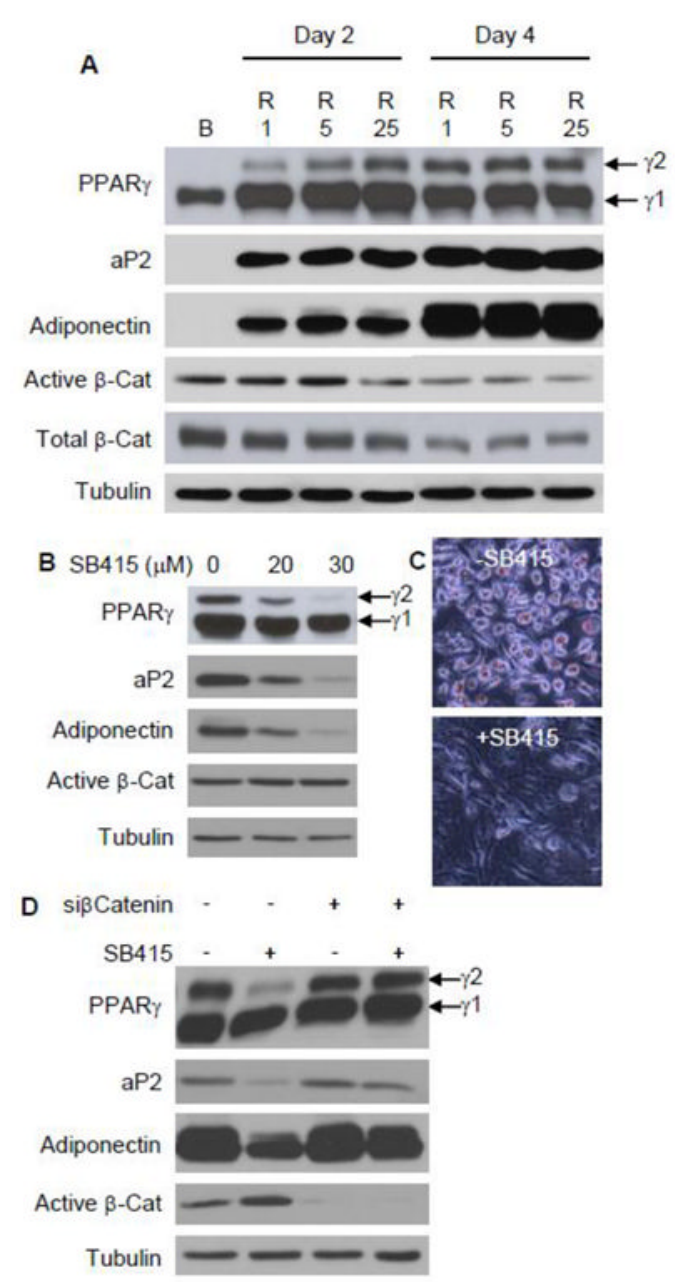

Fig. 1. GSK3 $\beta$ inhibition disrupts rosiglitazone-directed adipogenesis (A) mdMSC were cultured in adipogenic medium containing $5 \mu \mathrm{g} / \mathrm{ml}$ insulin, $0.1 \mu \mathrm{M}$ dexamethasone, and rosiglitazone $(\mathrm{R})$ at 1,5 , or $25 \mu \mathrm{M}$ and compared to mdMSC cultured in basal medium only (B) for $4 \mathrm{~d}$. Total cellular proteins were immunoblotted for designated proteins on the indicated days, with $\beta$-tubulin used as a loading control. (B) The GSK3 $\beta$ inhibitor SB415286 (SB415) was added to mdMSC in adipogenic medium with $5 \mu \mathrm{M}$ rosiglitazone and proteins were analyzed by immunoblotting on day 3. (C) Oil red $\mathrm{O}$ staining after treatment as in (B) for $4 \mathrm{~d}$. (D) Immunoblots of total cellular proteins from mdMSC transfected with nonsense siRNA (- siCat) or siRNA targeting $\beta$-catenin $(+$ siCat), then cultured in adipogenic medium with $1 \mu \mathrm{M}$ rosiglitazone $\pm 30 \mu \mathrm{M}$ SB415286 for $3 \mathrm{~d}$. 
A
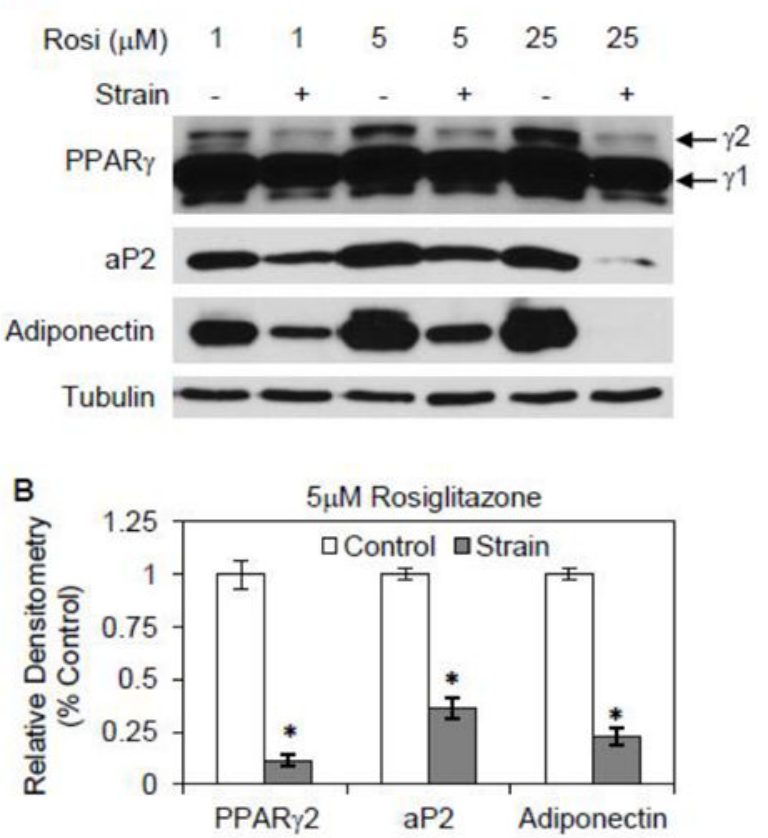

Normalized to $\beta$-tubulin

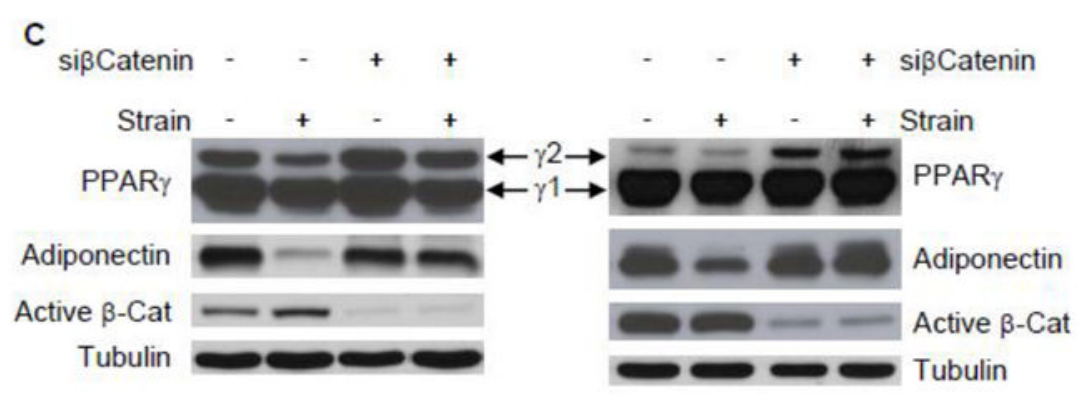

Fig. 2. Mechanical strain inhibits adipogenesis induced by rosiglitazone

(A) mdMSC in adipogenic medium containing rosiglitazone (Rosi) at 1, 5, or $25 \mu \mathrm{M}$ were exposed to a daily strain regimen $\times 3 \mathrm{~d}$, and total cellular proteins were immunoblotted for designated proteins. (B) Densitometric analyses of PPAR $\gamma 2$, aP2, and adiponectin were performed ( $\mathrm{n}=3$ experiments) after treatment as in (A) with adipogenic medium containing $5 \mu \mathrm{M}$ rosiglitazone. $* \mathrm{p}<0.001$ shows significant difference from unstrained control. (C) Immunoblots of total cellular proteins from mdMSC transfected with nonsense siRNA (siCat) or siRNA targeting $\beta$-catenin $(+$ siCat $)$, then cultured in adipogenic medium with 1 $\mu \mathrm{M}$ rosiglitazone \pm daily strain for $3 \mathrm{~d}$. Blots from two different experiments are included. 

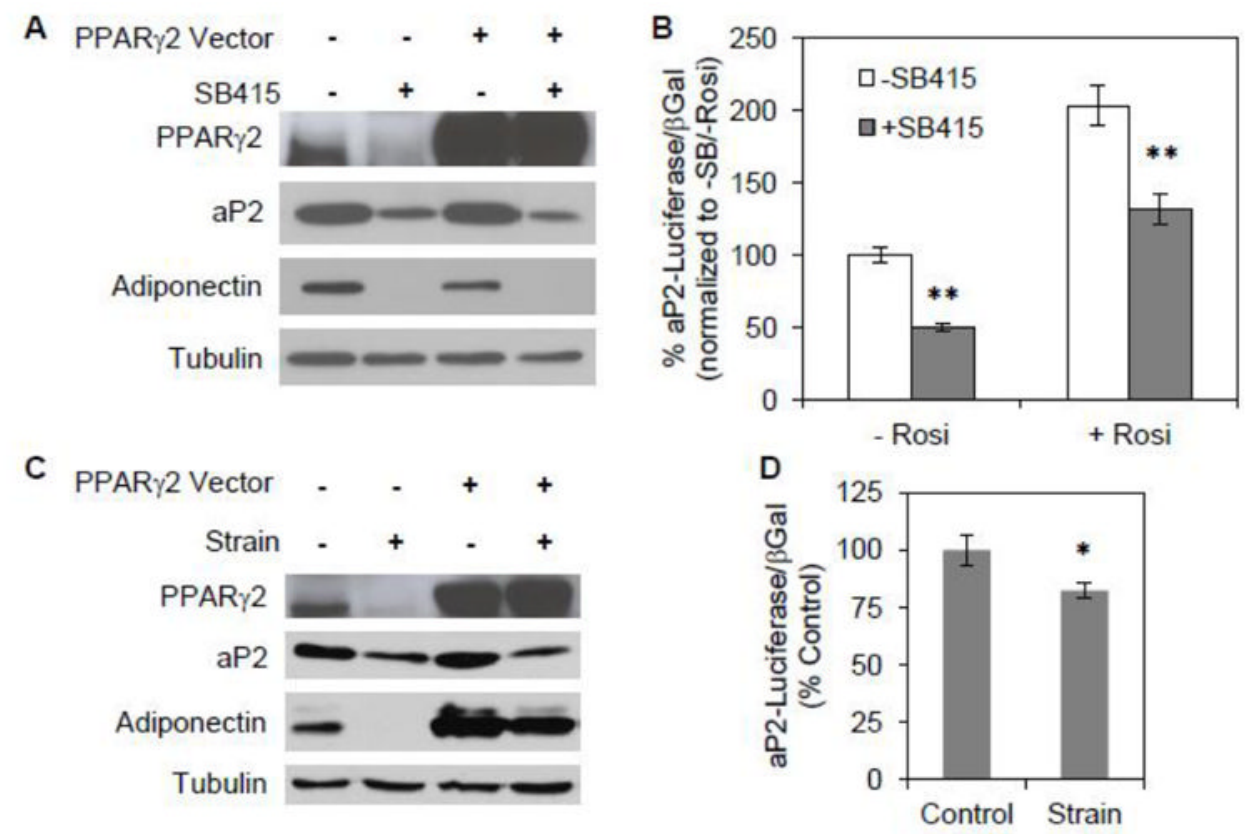

Fig. 3. Mechanical strain interferes with PPAR $\gamma$ function

(A) mdMSC were transfected with an overexpression vector for PPAR $\gamma 2$ or control empty vector. Adipogenic medium including rosiglitazone $\pm 30 \mu \mathrm{M} \mathrm{SB} 415286$ was added $24 \mathrm{~h}$ later and cells were analyzed at $3 \mathrm{~d}$ by immunoblot for designated proteins. (B) mdMSC were cotransfected with overexpression vectors for PPAR $\gamma 2$ and RXRa as well as an aP2-luciferase (aP2-luc) vector. $\beta$-galactosidase was used to control for transfection efficiency. The GSK3 $\beta$ inhibitor SB415286 (SB415) was added to basal medium (- Rosi) or medium including 1 $\mu \mathrm{M}$ rosiglitazone (+ Rosi) $24 \mathrm{~h}$ after transfection. aP2-luc reporter assay was performed $2 \mathrm{~d}$ later. Data were compiled from three experiments. $* * \mathrm{p}<0.001$ shows significant difference from medium without SB415. (C) mdMSC overexpressing PPAR $\gamma 2$ were cultured in adipogenic medium including rosiglitazone \pm daily strain for $3 \mathrm{~d}$ and proteins analyzed by immunoblotting. (D) mdMSC were co-transfected with PPAR $\gamma 2$ and RXRa, as well as aP2luc vector and $\beta$-galactosidase. Strain was applied concurrent with transfection. $24 \mathrm{~h}$ after transfection mdMSC were switched to medium containing $1 \mu \mathrm{M}$ rosiglitazone and a daily strain regimen was applied. aP2-luc reporter assay was performed $2 \mathrm{~d}$ later. Data were compiled from three experiments. $* \mathrm{p}<0.05$ shows significant difference from unstrained control. 

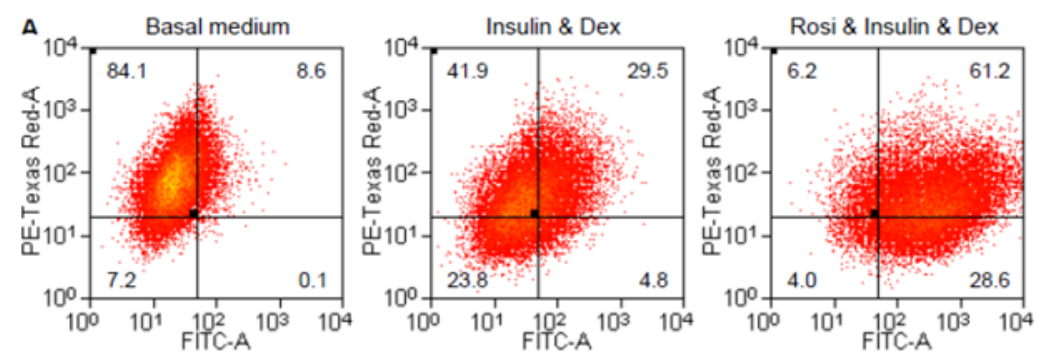

B
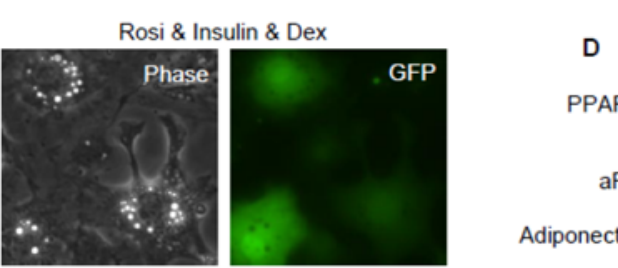

D

B $\frac{\text { Rosi }}{d 2 d 4}$
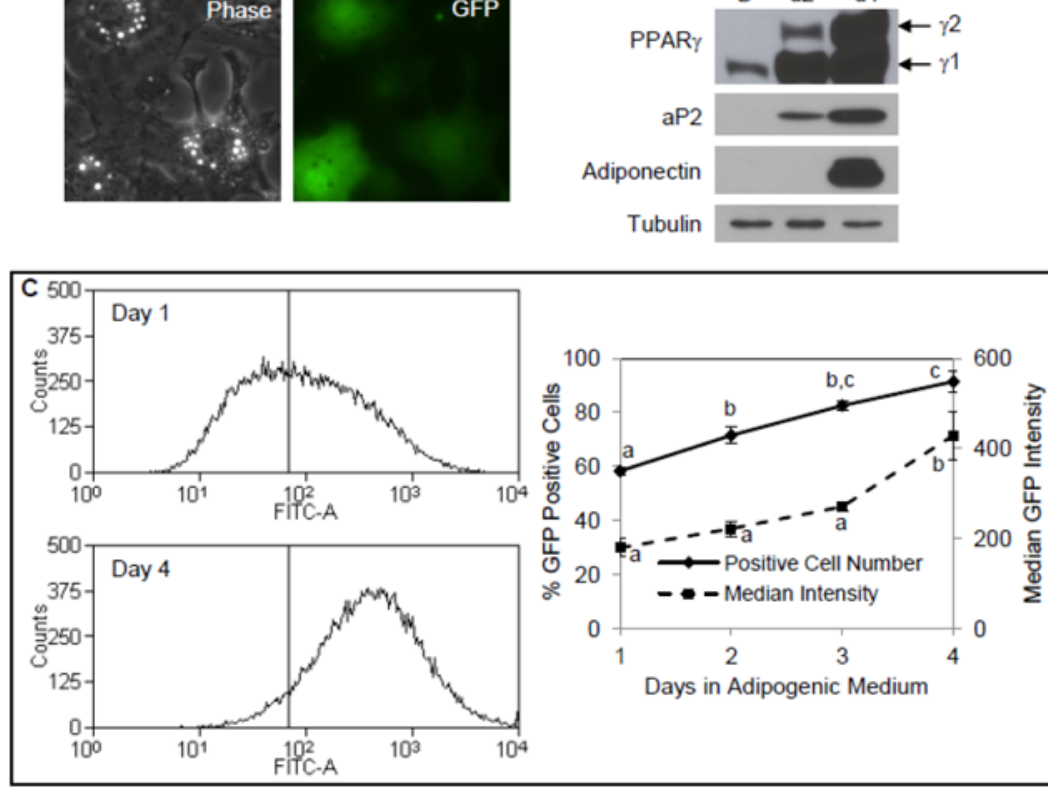

Fig. 4. Evaluating adipogenesis in individual cells using an aP2-GFP reporter mdMSC contained a GFPtopaz reporter for the aP2 promoter and a mCherry reporter for the SMaA promoter. (A) Cells were cultured in designated medium for $3 \mathrm{~d}$ prior to analysis by flow cytometry. Dex = dexamethasone; Rosi = rosiglitazone. (B) Fluorescent microscopy showing expression of aP2-GFP in individual cells after $4 \mathrm{~d}$ in adipogenic medium containing rosiglitazone, with a phase contrast image included to indicate cellular morphology. (C) Histograms (left) for aP2-GFP expression after 1 and $4 \mathrm{~d}$ in adipogenic medium. Vertical line indicates threshold for FITC intensity corresponding to positive GFP expression. Graph (right) showing positive GFP cell fraction and median GFP intensity versus days in adipogenic medium. Three experiments grouped for statistical analyses are shown in the graph. Days not sharing a letter are significantly different $(\mathrm{p}<0.05)$. (D) Immunoblots of total cellular proteins from cultures in adipogenic medium including rosiglitazone for 2 or $4 \mathrm{~d}$ compared to culture in basal (B) medium only. 

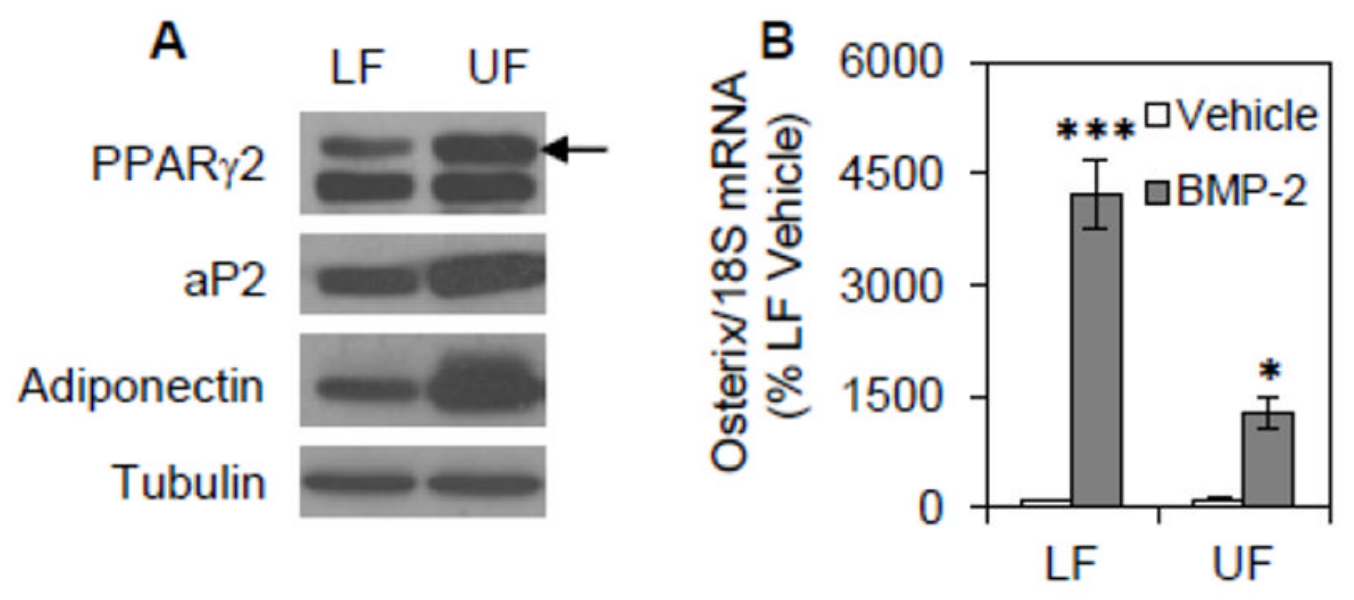

Fig. 5. Sorting by GFP intensity identifies a committed adipogenic population mdMSC containing the aP2-GFP construct were cultured in adipogenic medium including 5 $\mu \mathrm{M}$ rosiglitazone for $3 \mathrm{~d}$ then sorted into two subpopulations based upon GFP intensity. The subpopulation with the highest GFP intensity (top quintile of total cell population; defined as the upper fraction or "UF") was separated from the remainder of the cell population (defined as the lower fraction or "LF"). (A) Immunoblots of total cellular proteins isolated from each subpopulation. (B) The sorted subpopulations were re-plated into adherent culture. On the following day, cultures were treated with $125 \mathrm{ng} / \mathrm{ml}$ BMP-2 for $4 \mathrm{~d}$. Designated mRNA was amplified by real-time RT-PCR. Representative data are shown as mean \pm SEM and the experiment was repeated two times. ${ }^{*} \mathrm{p}<0.05$, *** $\mathrm{p}<0.001$ shows significant difference from vehicle. 
A

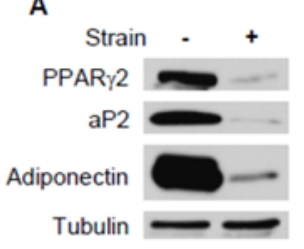

B

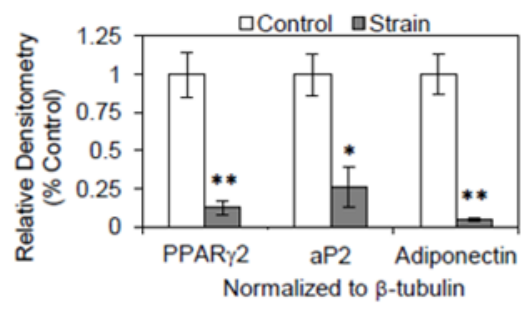

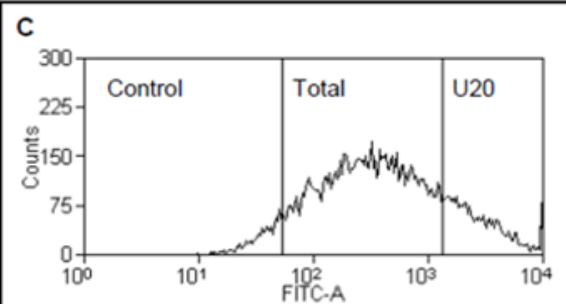
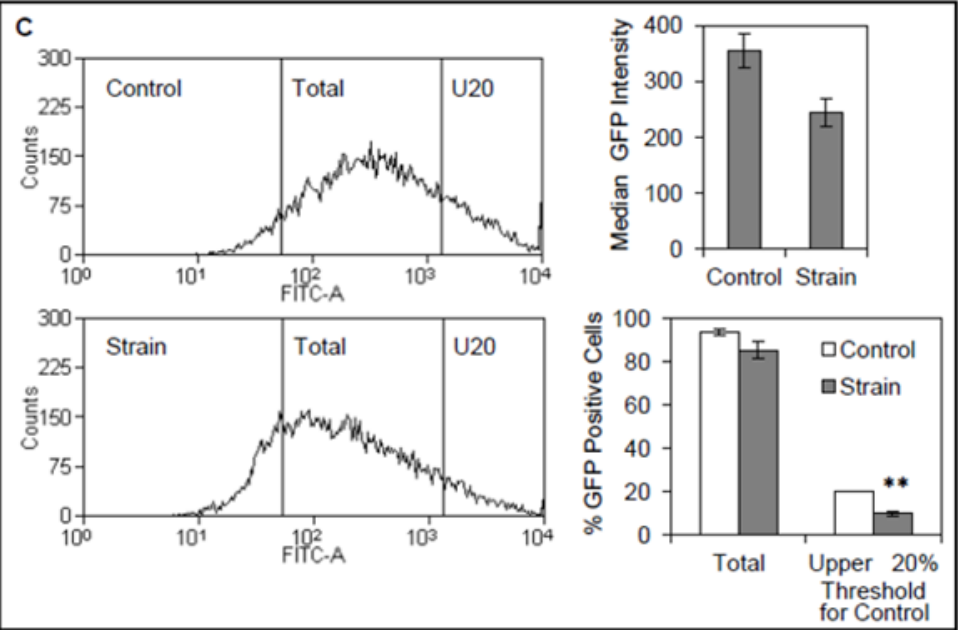

Fig. 6. Strain limits a committed adipogenic population induced by rosiglitazone (A) mdMSC containing the aP2-GFP construct were cultured in adipogenic medium containing $5 \mu \mathrm{M}$ rosiglitazone and exposed to a daily strain regimen $\times 4 \mathrm{~d}$. Total cellular proteins were immunoblotted for designated proteins. (B) Densitometric analyses of PPAR $\gamma 2$, aP2, and adiponectin were performed $(n=3$ experiments $)$ after treatment as in (A). $* \mathrm{p}<0.05, * * \mathrm{p}<0.01$ shows significant difference from unstrained control. (C) mdMSC treated as in (A) were evaluated by flow analysis. Histograms (left) for aP2-GFP expression in unstrained, control cultures or cultures subjected to a daily strain regimen. Left vertical line indicates threshold for FITC intensity corresponding to positive GFP expression and right vertical line (U20) indicates threshold for FITC intensity corresponding to upper quintile of GFP expression in control cultures. Histogram data were analyzed ( $\mathrm{n}=3$ experiments) for median GFP intensity (top right) and \% GFP positive cell fraction corresponding to each threshold level (bottom right). ${ }^{*} * \mathrm{p}<0.01$ shows significant difference from unstrained control. 

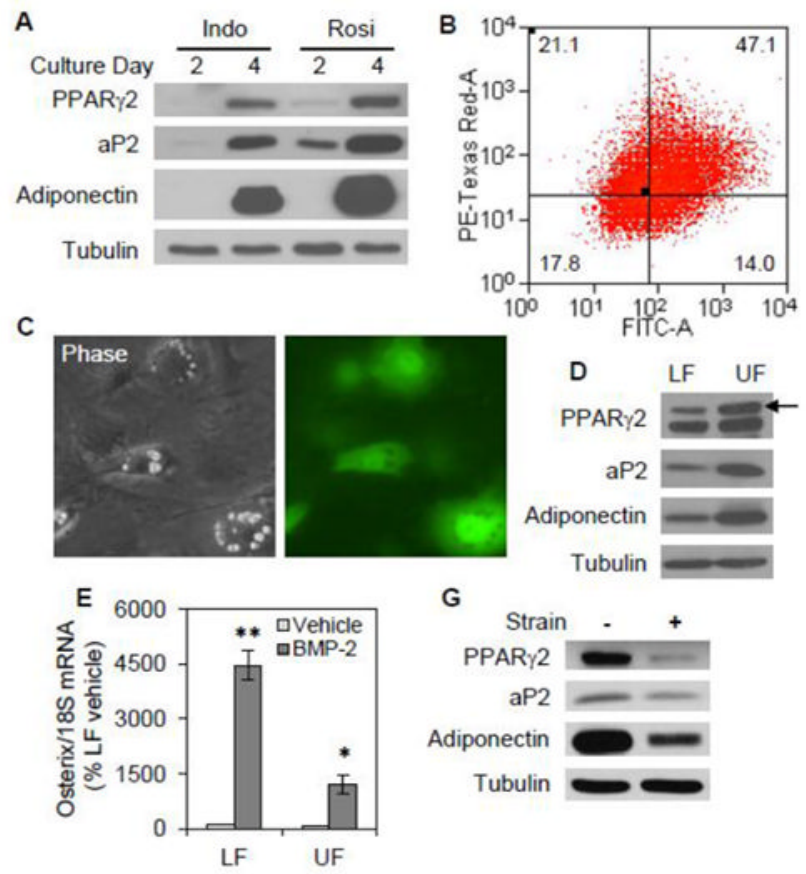

Tubulin
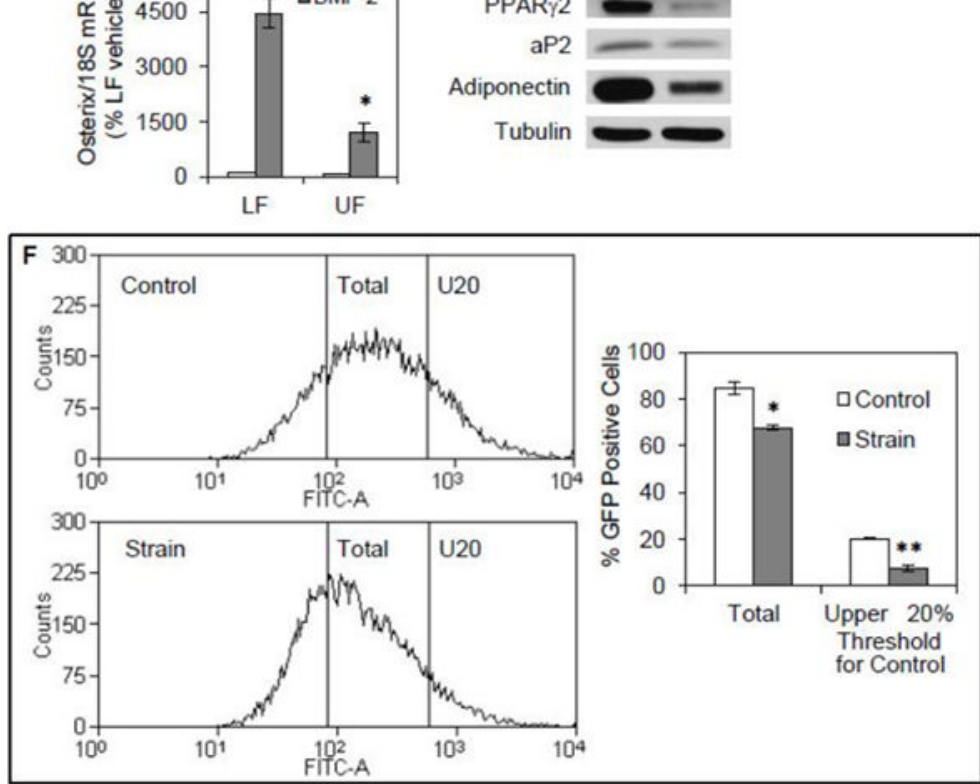

Fig. 7. Strain limits a committed adipogenic population induced by indomethacin mdMSC containing the aP2-GFP construct were cultured in adipogenic medium containing insulin, dexamethasone, and $50 \mu \mathrm{M}$ indomethacin (Indo). (A) Immunoblots of total cellular proteins from mdMSC cultured in adipogenic medium including Indo or $5 \mu \mathrm{M}$ rosiglitazone (Rosi) for 2 or 4 d. (B) Flow analysis of day 3 cultures. (C) Fluorescent microscopy showing expression of aP2-GFP in individual cells after $4 \mathrm{~d}$ in adipogenic medium containing inndomethacin, with a phase contrast image included to indicate cellular morphology. (D) mdMSC were cultured in adipogenic medium including indomethacin for 4d, and then sorted into two subpopulations based upon GFP intensity as described in figure 5. Immunoblots of total cellular proteins isolated from the upper fraction (UF) and the lower fraction (LF). (E) The sorted subpopulations were re-plated into adherent culture, and then treated with $125 \mathrm{ng} / \mathrm{ml}$ BMP-2 for $2 \mathrm{~d}$. Designated mRNA was amplified by real-time RTPCR. Data were compiled from two experiments. $* \mathrm{p}<0.01$, ** $\mathrm{p}<0.001$ shows significant difference from vehicle. $(\mathrm{F}) \mathrm{mdMSC}$ in adipogenic medium were exposed to a daily strain regimen $\times 5 \mathrm{~d}$ and evaluated by flow analysis. Histograms (left) for aP2-GFP expression in unstrained, control cultures or cultures subjected to a daily strain regimen. Left vertical line 
indicates threshold for FITC intensity corresponding to positive GFP expression and right vertical line (U20) indicates threshold for FITC intensity corresponding to upper quintile of GFP expression in control cultures. Histogram data $(\mathrm{n}=3$ experiments) were analyzed for $\%$ GFP positive cell fraction corresponding to each threshold level (right). * $<<0.01$, * $\mathrm{p}<0.001$ shows significant difference from unstrained control. (G) Immunoblots of total cellular proteins from mdMSC treated as in $(\mathrm{F})$. 